\title{
Measurement of contractile forces generated by individual fibroblasts on self-standing fiber scaffolds
}

\author{
Hojeong Jeon • Eunpa Kim • Costas P. Grigoropoulos
}

Published online: 23 September 2010

(C) The Author(s) 2010. This article is published with open access at Springerlink.com

\begin{abstract}
Contractility of cells in wound site is important to understand pathological wound healing and develop therapeutic strategies. In particular, contractile force generated by cells is a basic element for designing artificial three-dimensional cell culture scaffolds. Direct assessment of deformation of three-dimensional structured materials has been used to calculate contractile forces by averaging total forces with respect to the cell population number. However, macroscopic methods have offered only lower bounds of contractility due to experimental assumptions and the large variance of the spatial and temporal cell response. In the present study, cell contractility was examined microscopically in order to measure contractile forces generated by individual cells on self-standing fiber scaffolds that were fabricated via femtosecond laser-induced two-photon polymerization. Experimental assumptions and calculation errors that arose in previous studies of macroscopic and microscopic contractile force measurements could be reduced by adopting a columnar buckling model on individual, standing fiber scaffolds. Via quantifying eccentric critical loads for the buckling of fibers with various diameters, contractile forces of single cells were calculated in the range between $30-116 \mathrm{nN}$. In the present study, a force magnitude of approximately $200 \mathrm{nN}$ is suggested as upper bound of the contractile force exerted by single cells. In addition, contractile forces by multiple cells on a single fiber were calculated in the range between $241-709 \mathrm{nN}$.
\end{abstract}

H. Jeon · E. Kim • C. P. Grigoropoulos $(\bowtie)$

Laser Thermal Laboratory,

Department of Mechanical Engineering, University of California, Berkeley, CA 94720-1740, USA

e-mail: cgrigoro@me.berkeley.edu
Keywords Contraction · Fibroblast · Fiber scaffolds . Column buckling $\cdot$ Laser microfabrication $\cdot$ Two-photon polymerization

\section{Introduction}

Tractional and contractile forces generated by fibroblastic cells play a significant role in wound contraction and closure during the healing of an open wound. After cutaneous wounding, fibroblasts migrate to the fibronectin-fibrin wound interface and fill the wound, resulting in the formation of granulation tissue (Grinnell 1994; Tomasek et al. 2002). Tractional forces due to the migration of the fibroblasts reorganize the cells along stress lines to mechanically induce production of collagen and acquisition of proto-myofibroblast phenotype (Tomasek et al. 2002). In addition, tractional forces exerted by migrating cells at the defect edge are known to initiate wound contraction (Yannas 2001). These cells can be stimulated by tension and growth factors (TGF- $\beta$ ), to be differentiated to myofibroblasts that have $\alpha$-smooth-muscle actin microfilaments and generate increased contractile forces (Tomasek et al. 2002). Undifferentiated fibroblasts (fibroblasts and proto-myofibroblasts) also contribute to wound contraction, especially over the initial wound closure, although differentiated fibroblasts (myofibroblasts) play major role in generating contractile forces in defects (Tomasek et al. 2002; Yannas 2001).

A number of studies have been conducted by many investigators to establish the interaction between cell and extra cellular matrix (ECM) to unveil the mechanism of tissue contractions. Focal adhesions (FAs) are known to be capable of providing strong cell adhesion to ECM and are regions of signal transduction regulating cell growth (Burridge and Chrzanowska-Wodnicka 1996). Mechanical tension sensed 
through the FAs contributes to formation of stress fibers that transmit contractile forces (Tomasek et al. 2002; Burridge and Chrzanowska-Wodnicka 1996). The contractile forces of stress fibers are regulated by two kinase systems: myosin light chain kinase (MLCK) generating rapid contracting and Rho-kinase maintaining a sustained contraction (Katoh et al. 2001). Consequently, cells can generate tractional forces to the substrate during spreading and migrating (Burridge and Chrzanowska-Wodnicka 1996). Tractional forces were visualized by placing fibroblasts on thin distortable sheets of silicone rubber (Harris et al. 1981).

After the first visualization by Harris et al., the tractional and contractile forces have been visualized and detected by many different approaches. Flexible materials, including thin silicone rubber sheets, have been widely used in these experiments (Beningo and Wang 2002; Addae-Mensah and Wikswo 2008). The deformable substrates were combined with micropatterning and fluorescence imaging of FAs and used to measure tractional forces of myocytes and fibroblasts (Balaban et al. 2001). These methods require sophisticated computational analysis since the deformation exerted by cells tends to spread on continuous substrates. Hence, instead of uniformly flexible substrates, micromachined cantilevers were used to determine the subcellular forces generated by individual adhesive contacts by Galbraith and Sheetz (Galbraith and Sheetz 1997). Microfabricated arrays of microneedle-like posts were introduced by Tan et al. (Tan et al. 2003), removing the complexity incurred by continuous and extensive substrates with simplifying assumptions since distinct tractional forces could be measured. Such techniques can measure tractional forces from isolated cells and subcellular forces during cell spreading and migration. However, they are potentially limited in investigating three-dimensional tissues and interaction between cells and ECM. Experiments with tailored biomimetic materials such as collagen hydrogels and scaffolds have been studied to gain further understanding (Kolodney and Wysolmerski 1992; Delvoye et al. 1991; Freyman, et al. 2001; Grinnell 2000; Zahalak et al. 2000). In these studies, typically millions of cells were seeded on three-dimensional structures and contractile forces generated by the cells were measured and monitored as a function of time during cell culture (Kolodney and Wysolmerski 1992; Delvoye et al. 1991; Freyman et al. 2001). Contractile forces by single cells could be calculated by normalizing with respect to the total number of cells (Freyman et al. 2001; Zahalak et al. 2000). However, derivation of microscopic properties from deformation of macroscopic substrates may involve complicated assumptions that render this exercise difficult since cells are not contracting in the same direction and at the same time. Recently, a single strut from three-dimensional collagen-gel was used to measure contractile forces of individual cells
(Harley et al. 2007a). This novel method could estimate contractile forces of single cells more accurately than previous studies due to fewer experimental assumptions (Harley et al. 2007a). However, it is limited in controlling the diameter of the struts and producing an accurate account of the eccentricity of the cell loading applied to the struts.

To eliminate limitations encountered in previous measurements, we employed a femtosecond laser to fabricate polymer fiber scaffolds that served to measure contractile forces of individual cells. In our previous study, we developed a two-photon induced polymerization method to fabricate self-standing polymer fiber scaffolds and observed cell morphologies (Hidai et al. 2008, 2009). In the present work, we further used a femtosecond laser scissor to cut fibers, leaving them with one side clamped and the other free. The applied laser power controls the fiber scaffold diameter (Hidai et al. 2009) and henceforth the fiber mechanical strength. Contractile forces exerted by a single cell loaded on the fiber were calculated using buckling theory for an ideal pin-ended column. Therefore, isolated, standing fibers can yield more accurate forces over a wide range. By observing the buckling behavior of fibers during cell adhesion, spreading, and contraction, we could measure contractile forces and reasonably estimate the contractility of individual cells on three-dimensional scaffolds.

\section{Materials and methods}

\subsection{Fabrication of standing fiber scaffolds}

The fabrication of polymer fiber scaffolds via two-photon polymerization induced by femtosecond laser irradiation has been described in our previous studies (Hidai et al. 2008, 2009). A UV curable organic-inorganic hybrid polymer (ORMOCER ${ }^{\circledR}$, US-S4, Micro resist technology) was filled in between two glass plates $(25 \mathrm{~mm}$ in length, $3 \mathrm{~mm}$ in width, and $1 \mathrm{~mm}$ in thickness) that were assembled with $0.33 \mathrm{~mm}$-thick spacers. By irradiating a highrepetition-rate femtosecond laser beam (pulse width: $<500 \mathrm{fs}$, repetition rate: $1 \mathrm{MHz}$, wavelength: $1,045 \mathrm{~nm}$, FCPA $\mu$ Jewel D-400, IMRA America, inc.) into the uncured resin, two-photon polymerization occurred, and fibers were drawn along the laser beam path. The femtosecond laser beam was frequency doubled to the wavelength of $\sim 523 \mathrm{~nm}$ and focused at $\sim 500 \mu \mathrm{m}$ below the bottom glass plate/polymer resin interface with $5 \times$ microscope objective $(\mathrm{M}$ Plan Apo, N.A. $=0.14$, Mitutoyo). The exposure duration was controlled by a mechanical shutter and set at $0.2 \mathrm{~s}$. The samples were placed on $x-y$ axis motorized stages that were controlled by a PC. Upon conclusion of the polymerization process, the 
samples were placed on a hot plate at $110^{\circ} \mathrm{C}$ for $10 \mathrm{~min}$, and afterwards uncured resin was removed in a developer (ORMODEV ${ }^{\circledR}$, Micro resist technology) for $30 \mathrm{~min}$. The sample was then rinsed three times with iso-propanol and deionized water with $60 \mathrm{mg} / \mathrm{mL}$ asolectin (Sigma Aldrich). The suspended fiber scaffolds were dipped in $70 \%$ ethanol for sterilization, exposed for coating to $20 \mu \mathrm{g} / \mathrm{mL}$ fibronectin (Sigma Aldrich) in phosphate buffered saline (PBS, Gibco Invitrogen) for $1 \mathrm{~h}$, and rinsed with PBS three times. Finally, they were severed using amplified femtosecond laser pulses (pulse width: $\sim 100 \mathrm{fs}$, repetition rate: $1 \mathrm{kHz}$, wavelength: $800 \mathrm{~nm}$, Spitfire, Newport, Irvine, CA) with $10 \times$ microscope objective (M Plan Apo, N.A. $=0.25$, Mitutoyo) to set the length of the cantilever fibers at $\sim 200 \mu \mathrm{m}$.

\subsection{Characterization of the fiber scaffolds}

Through the fabrication process mentioned above, the polymer fibers were $\sim 200 \mu \mathrm{m}$ in length, with one end clamped and the other free. The fiber diameter $(\sim 5-$ $20 \mu \mathrm{m}$ ) could be controlled by adjusting laser power and irradiation time (Hidai et al. 2009). The diameters were measured from optical transmitted images taken via a $40 \times$ microscope objective (LUCPlanFLN, N.A. $=0.6$, Olympus) on an inverted microscope (IX71, Olympus) before cell culturing.

The elastic modulus $\left(E_{s}\right)$ of the fiber scaffolds was measured via atomic force microscopy (AFM, XE-100, Park Systems) with a tip-less cantilever (TL-CONT-SPL, Nanosensors). The force constant of the cantilever was measured by a thermal tuning method (Hutter and Bechhoefer 1993) and calculated to be $0.0363 \mathrm{~N} / \mathrm{m}$ using AFM software (XEI, Park Systems). A fiber with $200-\mu \mathrm{m}$ length $(l)$ and $20-\mu \mathrm{m}$ diameter $(D)$ was chosen as reference. The fiber sample was placed on a plastic dish filled with water (Fig. 1). The fibers are of circular cross section. Therefore, the modulus was obtained from Eq. 1 with the cantilever displacement $\left(d_{c}\right)$, the force constant of the cantilever $\left(K_{c}\right)$, the relative deflection of the free end of the fiber $\left(d_{f}\right)$, and moment of inertia for a cylinder (Harley et al. 2007b).

$E_{s}=\frac{64 K_{c}\left(d_{c}-d_{f}\right) l^{3}}{3 \pi D^{4} d_{f}}$

The bending test was performed independently five times to yield an average value of the modulus.

\subsection{Calculation of contractile forces}

When a cell grows on the fiber, an axial compressive force $(P)$ is generated by the cell contraction. If the contractile force is larger than the critical load $\left(P_{c r}\right)$, buckling of the fiber occurs. The critical buckling load generated by an (a)

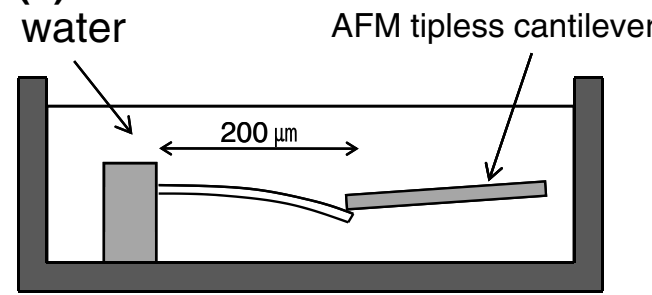

(b)

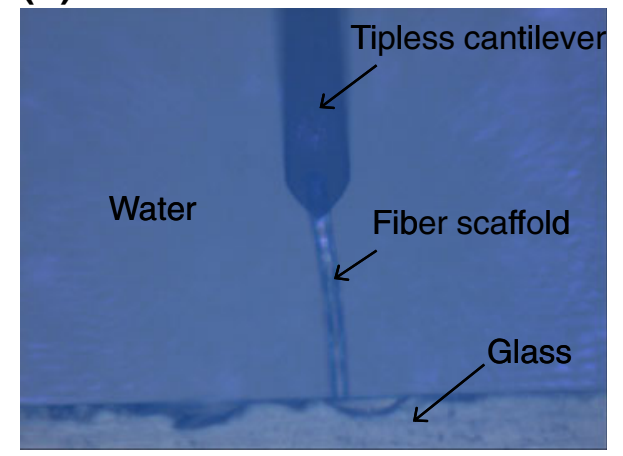

Fig. 1 Schematic of bending test with fiber scaffolds (a) and a top view of a real optical image (b)

individual cell attached on the fiber as displayed schematically in Fig. 2 was calculated by the Euler buckling load formula (Craig 1996):

$I=\frac{\pi D^{4}}{64}$

$P_{c r}=\frac{\pi^{2} E_{S} I}{L_{e f f}^{2}}$

where $I$ signifies the moment of inertia for a cylinder, and $L$ and $D$ denote the length and diameter of the fiber,

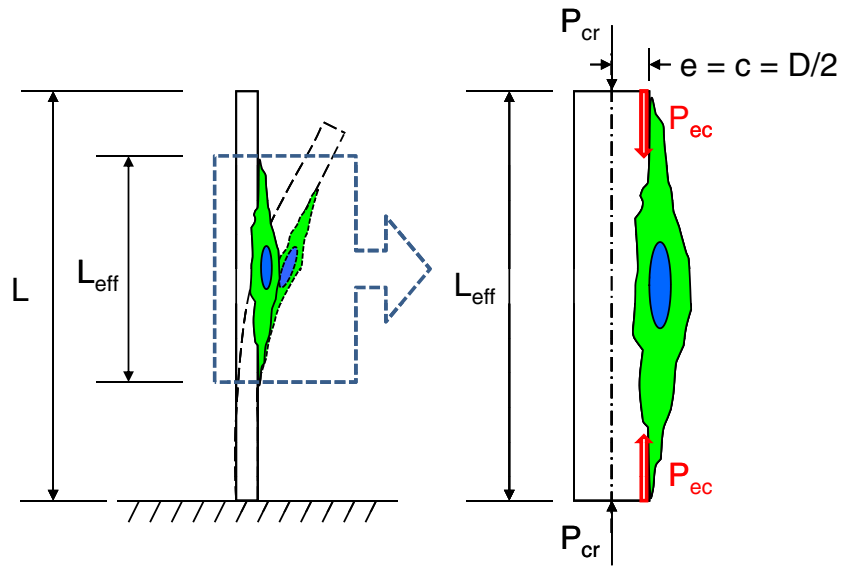

(a)

(b)

Fig. 2 Schematic of buckling of a fiber scaffold by a contractile force generated by a single cell. Once a cell attached and spread on a fiber, the fiber started to be buckled (a). The total forces to buckle the fiber were considered to act at the end of the cell and on the surface of the fiber (b) 
respectively. In this study, $L_{\text {eff }}$ was defined as the length of a single cell on a fiber. The length of a single cell on a fiber was taken at $\sim 100 \mu \mathrm{m}$, consistent with our previous work (Hidai et al. 2009). Because the cells attach and apply contractile forces on the surface of the fiber scaffolds, eccentricity should be considered when calculating the critical buckling load. Consequently, a secant formula given by Eq. 4 was used to calculate the contractile force (Craig 1996). The eccentric load $\left(P_{e c}\right)$ and critical load $\left(P_{c r}\right)$ are associated to the eccentricity $(e)$, the distance from the neutral axis to the fiber surface $(c)$, the cross-sectional area of the compression column $(A)$, and the radius of gyration $(r=\sqrt{I / A})$ :

$P_{c r}=P_{e c}\left[1+\left(\frac{e \times c}{r^{2}}\right) \sec \left(\frac{L_{e f f}}{2 r} \sqrt{\frac{P_{e c}}{A E_{s}}}\right)\right]$

In this calculation, $L_{\text {eff }}$ was also defined as $100 \mu \mathrm{m}$, and $e$ and $c$ were taken as the fiber radius. Substituting the critical load $\left(P_{c r}\right)$ calculated from the Eq. 3 into Eq. 4, the cell-generated contractile forces were calculated.

\subsection{Cell culture and imaging}

Fibroblasts, NIH3T3, were cultured in a $75-\mathrm{cm}^{2}$ culturing flask (Corning) containing Dulbecco's Modified Eagle Medium (Gibco Invitrogen), 10\% fetal bovine serum (FBS) (Gibco Invitrogen) and 100 units $/ \mathrm{ml}$ penicillin (Gibco Invitrogen), maintained at $37^{\circ} \mathrm{C}$ and $5 \% \mathrm{CO}_{2}$ culture incubator. Before conducting the experiments, confluent cells were detached from the flask by $0.05 \%$ Trypsin-EDTA (Gibco Invitrogen) and seeded onto the fiber scaffolds which were placed in sterile polystyrene well plates and maintained in $\mathrm{CO}_{2}$-independent media (Gibco Invitrogen), 10\% FBS, 100 units $/ \mathrm{ml}$ penicillin, and $1 \%$ GlutaMAX (Gibco Invitrogen). Cell mediated contraction was monitored via a $10 \times$ objective lens (Plan Achromat, N.A. $=0.25$, Olympus) with an inverted microscope (IX71, Olympus) with temperature maintained at $37^{\circ} \mathrm{C}$. Transmission images were taken every 1 min with a digital CCD camera (Retiga 2000R cooled, Qimaging) for about $400 \mathrm{~min}(\sim 7 \mathrm{~h})$. Cell migration or bridging was monitored by consecutive transmission images, and isolated cells were chosen to measure single cellmediated contractile forces.

For fluorescence images, cells on fibers suspended between two glasses (before cutting the fibers) were fixed with $3.7 \%$ formaldehyde (Fisher Scientific), and then permeabilized with $0.1 \%$ Triton X-100 (Fisher Scientific) after one day culturing. Actin cytoskeletons were stained with $330 \mathrm{nM}$ alexa fluor 488 phalloidin (Invitrogen) for $40 \mathrm{~min}$, and nuclei were stained with $300 \mathrm{nM}$ DAPI (Invitrogen) for $4 \mathrm{~min}$. The samples were kept in PBS and images were taken by a 510 Meta UV/VIS confocal microscope (Zeiss) with a $63 \times \mathrm{W}$ Plan Apochromat dipping objective lens (Zeiss).

\section{Results}

\subsection{Fiber fabrication and elastic modulus}

A UV curable polymer resin can be selectively cured within the beam depth of focus by femtosecond laser irradiation at an arbitrary position inside the resin due to two-photon absorption (Kawata et al. 2001). Using the two-photon polymerization, a fiber scaffold fabrication method has been reported (Hidai et al. 2008, 2009). In order to measure cell-mediated contractile force, fibers of $\sim 0.33 \mathrm{~mm}$ length and $\sim 50 \mu \mathrm{m}$ pitch were fabricated bridging two glass plates. The fiber diameters were controlled by changing the laser power with fixed exposure time of $0.2 \mathrm{~s}$ (Hidai et al. 2009). In this study, different diameters of fibers in the range of $\sim 5-\sim 15 \mu \mathrm{m}$ were fabricated for measuring contractile forces. Finally, an amplified Ti:sapphire femtosecond laser beam was used to cut free one end of the fibers.

Bending test was performed on fibers with $200-\mu \mathrm{m}$ length and $20-\mu \mathrm{m}$ diameter under water (Fig. 1) via AFM, and linear elastic behavior was observed (Fig. 3). The 20- $\mu \mathrm{m}$ diameter fiber was chosen in order to generate similar bending displacements of the fiber, $d_{f}$, and the AFM cantilever, $d_{c}-d_{f}$, (see Eq. 1) since accurate measurements cannot be achieved if the displacements, $d_{f}$ or $d_{c}-d_{f}$, are very small. Using Eq. 1 , the elastic modulus of the fiber was calculated to be $6.01 \pm 0.87 \mathrm{MPa}$. The bending test was also performed on dry fibers, but the results did not exhibit significant deviation from the submerged ones.

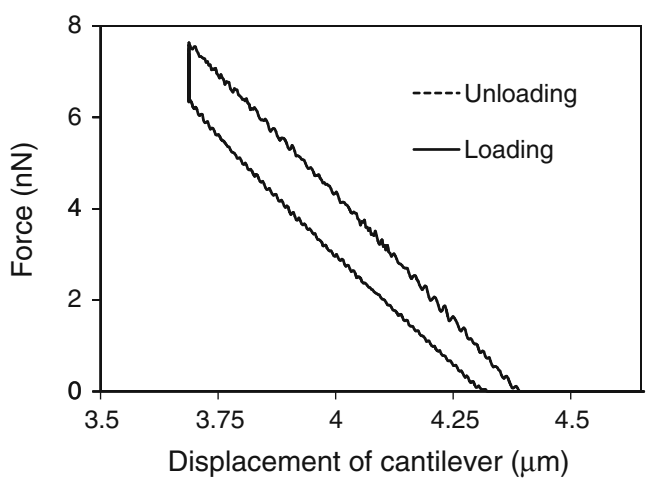

Fig. 3 Characteristic of load-unload bending experiment performed via AFM for a single fiber 


\subsection{Single cell-mediated contractile forces}

Typical morphologies of single and multiple cells on fibers were shown in Fig. 4. The cells were elongated and wrapped around the fiber forming shapes of high length/width ratio. In particular, single cells wrapped the fiber partially, and their actin stress fibers stretched on the fiber top surface (Fig. 4(a)). This is the most efficient configuration for inducing one-dimensionally distributed contractile forces on fiber scaffolds.

Single cell-produced contractile forces were obtained by calculating eccentric loads for buckling of a cylindrical column. By substituting the fiber elastic modulus into Eq. 3, the critical loads for buckling were calculated. Thereafter, the eccentric loads were derived upon substituting the critical loads into Eq. 4. Results for six different diameters are shown in Table 1. Figure 5(a) shows fiber scaffolds having six different diameters right after cell
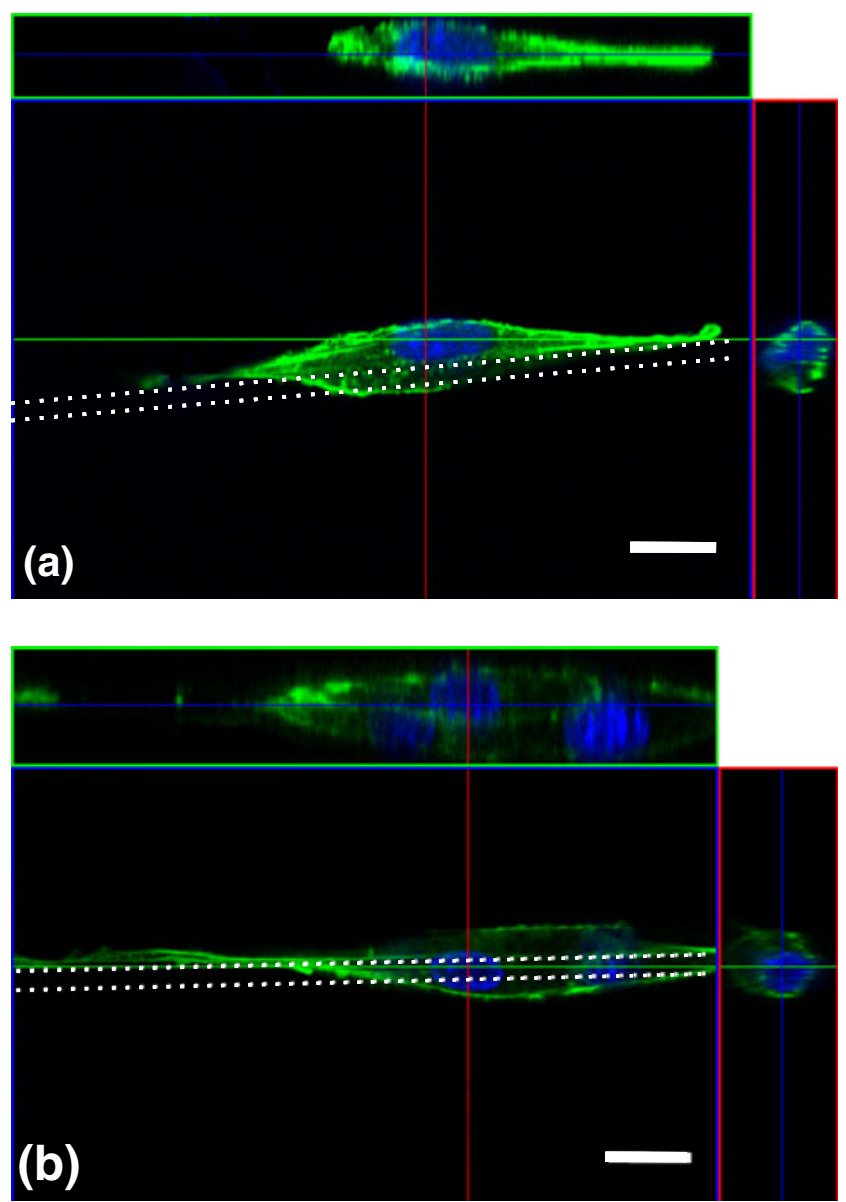

Fig. 4 Confocal fluorescence microscope images of threedimensional cell morphology and cross-sectional images of a single cell (a) and multiple cells (b). Stained actin cytoskeleton (Alexa 488phalloidin: green) and nuclei (DAPI: blue). White dotted lines indicate fiber scaffolds of $8 \mu \mathrm{m}$ in diameter. Scale bars: $20 \mu \mathrm{m}$
Table 1 Calculated critical loads and eccentric loads for fiber buckling depending on fiber diameters

\begin{tabular}{lcc}
\hline Diameter $(\mu \mathrm{m})$ & $P_{c r}(\mathrm{nN})$ & $P_{e c}(\mathrm{nN})$ \\
\hline 5 & 181 & 30 \\
6 & 376 & 63 \\
7 & 697 & 116 \\
8 & 1189 & 198 \\
8.4 & 1445 & 241 \\
11 & 4249 & 709 \\
\hline
\end{tabular}

loading. Some fibers were already bent due to pressure exerted by shock wave or bubble generated during the laser cutting process. Errors from such bent fibers were eliminated since buckling of similar diameter fibers by cell-mediated forces was verified through several inde-
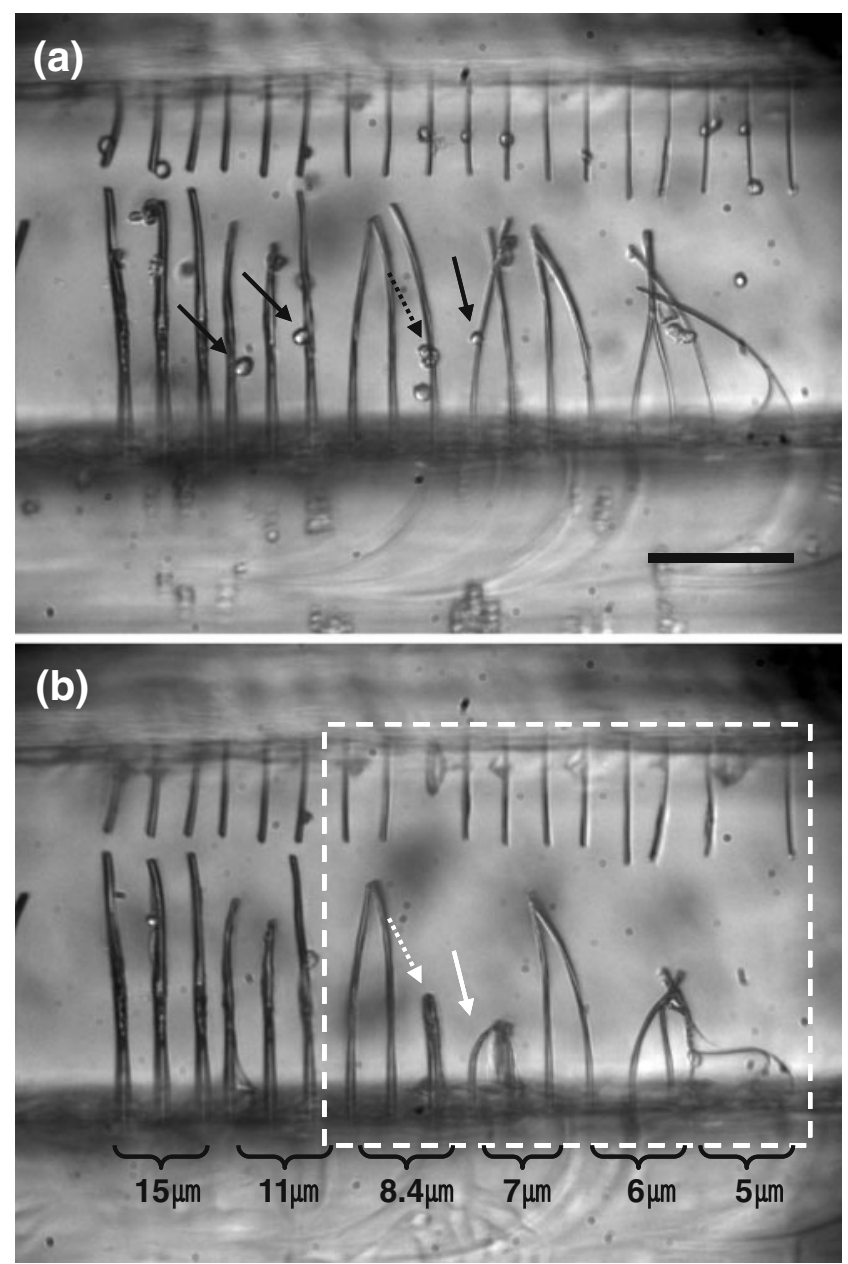

Fig. 5 Microscope images of fiber scaffolds right after cell loading (a) and 350 min after cell loading (b). Black-line arrows indicate cells right before cell adhesion, and white-line arrows indicate cells on buckled fibers. Solid-line arrows indicate single cells, and dashed-line arrows indicate multiple cells. The dashed-line box in (b) highlights buckled fibers. Scale bars: $200 \mu \mathrm{m}$ 
pendent experiments. Figure 5(b) was taken at an elapsed time of $350 \mathrm{~min}$ after cell loading. Fiber buckling was observed for the 5-, 6-, 7-, and 8.4- $\mu \mathrm{m}$ diameter fibers, although the $8.4-\mu \mathrm{m}$ diameter fiber was buckled by multiple cells. On the basis of more than five independent experiments, we concluded that individual cells could buckle $7-\mu \mathrm{m}$ or smaller diameter fibers, but not $8-\mu \mathrm{m}$ or thicker fibers. This suggests that the contractile forces generated by an individual cell are in the range of 30 $116 \mathrm{nN}$ that are the eccentric critical loads for $5-7-\mu \mathrm{m}$ diameter fibers. In addition, it should be noted that an upper bound of the single cell-mediated contractile force measured in this study is $\sim 200 \mathrm{nN}$ that is the eccentric critical load for $8-\mu \mathrm{m}$ diameter fibers. Therefore, buckling of thinner fibers occurs much faster than thicker fibers since the critical loads for buckling of thinner fibers are smaller than the maximum contractile forces of cells (Fig. 6 and Table 1). Buckling of fibers with diameter of $5-\mu \mathrm{m}$ started at $30 \mathrm{~min}$ after cell loading. In contrast, buckling could be observed at an elapsed time of $160 \mathrm{~min}$ for the 6- and 7- $\mu \mathrm{m}$ diameter fibers.
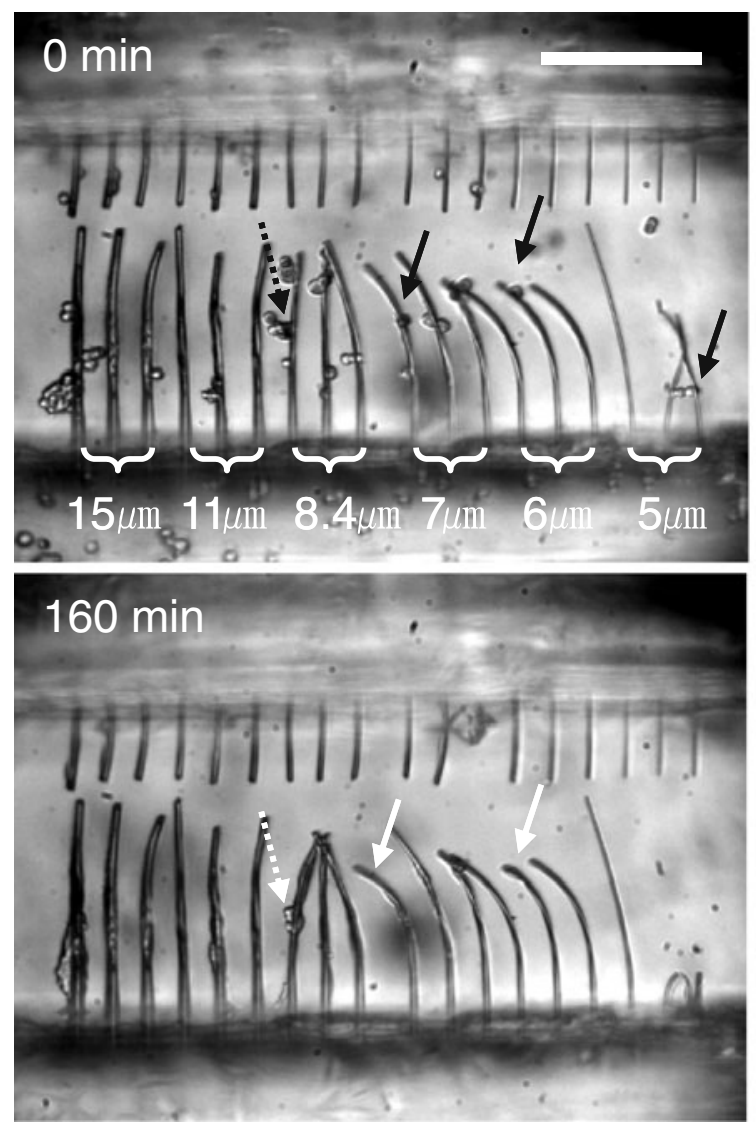

Fig. 6 Time-lapse microscope images of fiber buckling for different size fibers. White arrows represent the beginning points of buckling, and the numbers in the top-left corner indicate the time. Black arrows indicate

\subsection{Multiple cell-mediated contractile forces}

While individual cells could buckle fibers thinner than $8 \mu \mathrm{m}, 8.4-\mu \mathrm{m}$ diameter fibers buckled only upon loading by multiple cells. Thicker fibers with diameters of 11- and $15-\mu \mathrm{m}$ remained straight and intact (Figs. 5 and 6). The eccentric critical loads for the $8.4-\mu \mathrm{m}$ and $11-\mu \mathrm{m}$ fibers are 241 and $709 \mathrm{nN}$, respectively (Table 1). Since multiple cells tend to accumulate in a restricted area (shown in Fig. 4 (b)), the respective buckling loads were not linearly proportional to their number. Furthermore, the buckling load did not exceed $709 \mathrm{nN}$, i.e. the buckling load of $11-\mu \mathrm{m}$ diameter fiber. This suggests that the contractile forces generated by multiple cells on a single fiber lie between 241-709 $\mathrm{nN}$. The thicker fibers $(11$ and $15 \mu \mathrm{m})$ were not buckled by multiple cells, but rapid buckling of thinner fibers $(6 \mu \mathrm{m})$ by multiple cells was observed. Figure 7 shows time-lapse images of $6-\mu \mathrm{m}$ diameter fiber buckling induced by single and multiple cells. The fiber buckling by multiple cells (60 $\mathrm{min}$ ) occurred rapidly compared to individual cells (120-160 min). This result suggests that the contractile
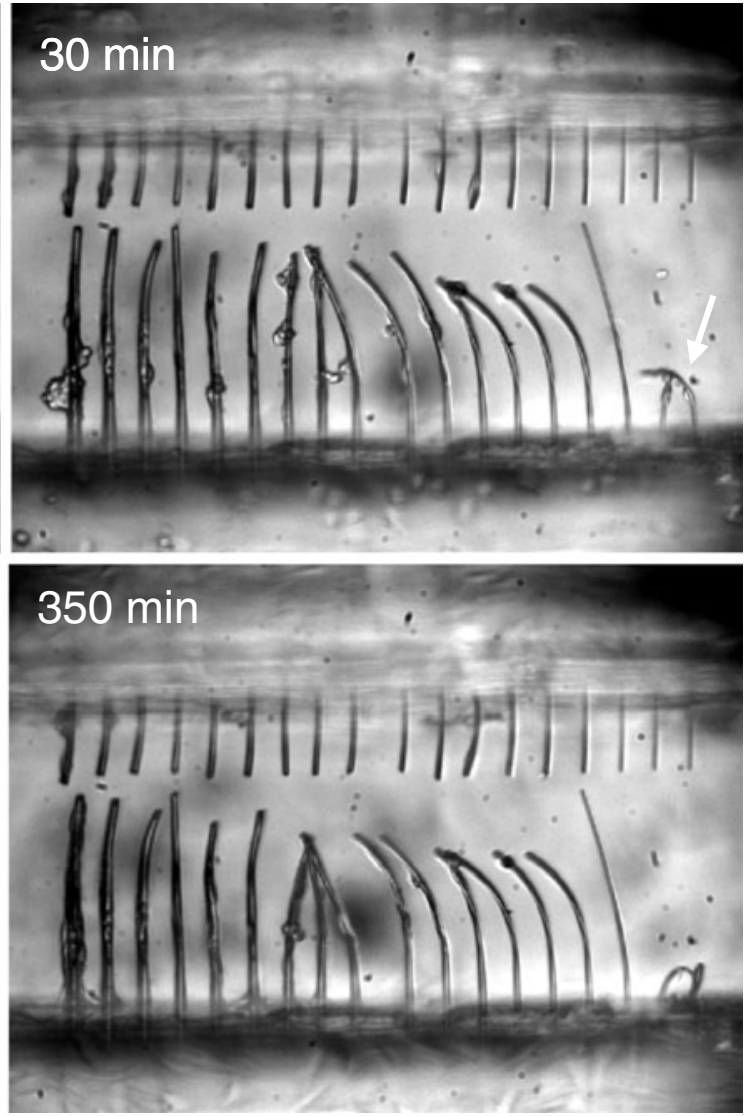

cells right before cell adhesion. Solid-line arrows indicate single cells, and dashed-line arrows indicate multiple cells. The numbers in top-left corners indicate the elapsed time. Scale bars: $200 \mu \mathrm{m}$ 

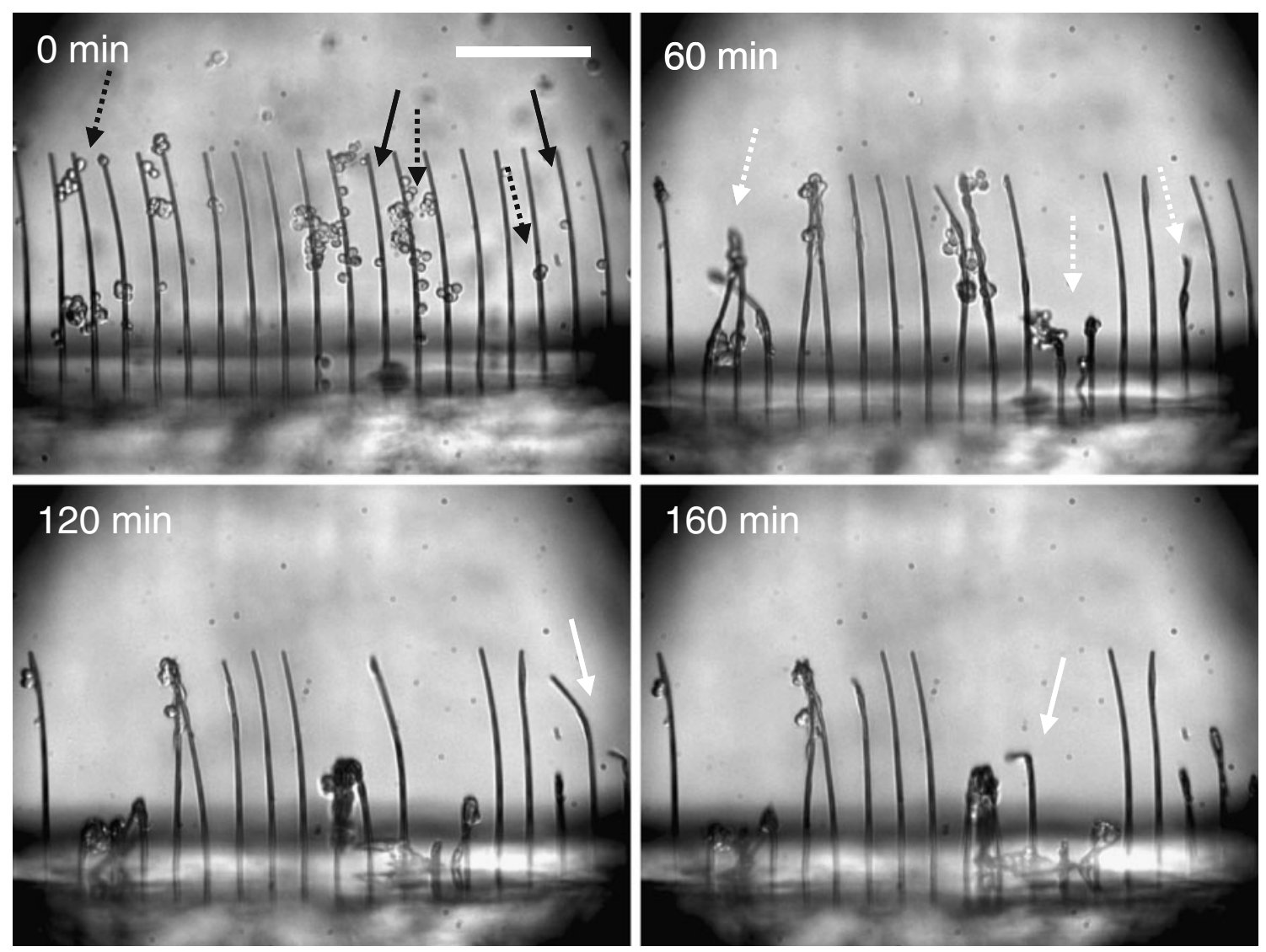

Fig. 7 Time-lapse microscope images of fiber buckling for $6-\mu \mathrm{m}$ fibers. Black-line arrows indicate cells right before cell adhesion. White dashed-line arrows indicate the beginning points of buckling by

multiple cells, and white solid-line arrows indicate the beginning points of buckling by single cells. The numbers in top-left corners indicate the elapsed time. Scale bars: $200 \mu \mathrm{m}$

forces generated by multiple cells are bigger than those by single cells, although the forces do not scale linearly proportional to the number of cells.

\section{Discussion}

We estimated individual cell mediated contractile forces by considering columnar buckling of cantilever fiber scaffolds. This procedure allows more accurate calculation due to reduced experimental assumptions compared to previous studies on two-dimensional continuous surfaces or pinarray detectors and three-dimensional hydrogel or scaffolds. Measurement of cell traction forces using two-dimensional flexible materials can yield the force distribution at specific focal adhesions within a single cell. However, it requires sophisticated analysis and does not reveal cell response to a fibrillar three-dimensional environment due to differences in cell morphology, cytoskeleton organization and focal adhesion strength (Beningo and Wang 2002; Harley et al. 2007a; Hidai et al. 2009). Measurement of the forces on three-dimensional hydrogel scaffolds is more directly connected to in vivo cellular microenvironment and tissue engineering. However, the respective macroscopic force involves significant assumptions: (1) cell density does not change during measurement; (2) all cells contribute equally to force generation at the same time; (3) the force direction is identical; and (4) the material is isotropic. Due to these assumptions, the forces obtained on three-dimensional materials can only provide lower bounds to single cell contractile forces. Recently, a new method for measuring individual cell mediated contractile forces using a single strut of collagen-glycosaminoglycan (CG) scaffolds was reported by Harley et al. This microscopic method relaxes experimental assumptions (Harley et al. 2007a), but still cannot produce accurate force measurement since it is limited to specific scaffold structure. To address these concerns, we used single-standing fiber columns with varying diameters to control mechanical properties of the fibers and measure the contractile forces exerted by individual cells. Because of the microscopic observation of the simple columnar fiber structure, the experimental assumptions and calculation errors can be reduced. Individual fibroblasts could deform fiber scaffolds that require 
up to $200 \mathrm{nN}$ of eccentric critical load for buckling. This value represents an upper bound of the contractile force generated by a single cell, that is lower than the upper bound force $(450 \mathrm{nN})$ suggested by Harley et al. (Harley et al. 2007a). Our results provide enhanced evidence in support of their measurement of contractile forces of individual cells, since they suggested an upper bound $(450 \mathrm{nN})$ without measuring forces in the intermediate range between $50 \mathrm{nN}$ and $450 \mathrm{nN}$.

The contractile force measurement is important since the forces generated by cells depend on cell morphology that is in turn influenced by the supporting three-dimensional material structure. It was reported that most of forces are generated by cells upon attachment and are associated with changes in cell shape (Eastwood et al. 1996). In addition, increased cell spreading enhances stress fiber formation and cytoskeletal tension, while the size of focal adhesions has a positive correlation to the forces (Tan et al. 2003). Fibroblast cellular morphology on a single fiber structure is much different from that on two-dimensional surfaces. In this regard, cells on a single fiber become elongated and wrap the fiber partially with a straight cytoskeleton shape (Hidai et al. 2009). Due to the one-dimensional, elongated cell morphology in the present study, the forces exerted by the cells do not disperse but can effectively buckle the fiber columns. The fiber buckling occurred on fibers smaller than $8.4 \mu \mathrm{m}$ in diameter. Thinner fibers were buckled earlier than thicker fibers; e.g. 5- $\mu \mathrm{m}$ diameter fibers buckled within $30 \mathrm{~min}$ after cell seeding, and the other fibers buckled within 120-160 min after cell seeding. The seeded cells attach to the fiber and start spreading within $30 \mathrm{~min}$. It is noted that traction force applying eccentric load during adhesion suffices for buckling the 5 - $\mu \mathrm{m}$ fibers. The eccentric critical load for the 5 - $\mu \mathrm{m}$ diameter fiber buckling is $30 \mathrm{nN}$ (Table 1). After the cells spread completely, thicker fibers buckled by cell locomotion and contraction. Previous studies of macroscopic measurement using collagen gel or scaffolds reported that the forces generated by seeded cells tend to increase rapidly in the early phase, reaching an asymptotic limit (Tomasek et al. 2002; Freyman et al. 2001; Eastwood et al. 1996). In the early stage of wound healing, traction forces generated by cell locomotion are sufficient for wound closure and stimulate fibroblasts to develop stress lines via application of mechanical stress (Tomasek et al. 2002; Ehrlich and Rajaratnam 1990). This study suggests that single cell-mediated forces in the early stage are $\sim 30-63 \mathrm{nN}$ within $\sim 30 \mathrm{~min}$ and the force increase up to $\sim 116-200 \mathrm{nN}$ within $\sim 3 \mathrm{~h}$ after cell seeding.

The effect of multiple cells in generating contractile forces was also investigated. Freyman et al. reported that contractile forces scaled with respect to the population of fibroblasts within a CG scaffold were linearly proportional to the number of cells and suggested that fibroblasts could exert forces independent of the presence of other cells (Freyman et al. 2001). In our study, however, the contractile force did not increase as the number of cells increased. Fibers of $8.4-\mu \mathrm{m}$ diameter deformed, but thicker fibers did not buckle, although they were deformed by cells suspended between adjacent fibers, in which case forces normal to the fiber axis were applied. A single fiber does not offer sufficient area to accommodate adhesion of multiple cells. As a result, cell-mediated forces do not apply on the same surface of the fibers. Consequently, contractile forces generated by multiple cells cannot induce buckling of single fibers effectively in this study.

The difference between microscopic and macroscopic measurement does not only refer to the multiple cell effect but also to the magnitude of the force generated by individual cells. Here, the fibers subjected to eccentric critical loads in the range of $30-116 \mathrm{nN}$ deformed easily. Such forces are not much different than the value of $41 \mathrm{nN}$ quoted by Harley et al. (Harley et al. 2007a). However, forces calculated by macroscopic measurements are of the order of $1 \mathrm{nN}$, which is much smaller than our and Harley et al.'s results (Freyman et al. 2001; Eastwood et al. 1996). Forces are not generated simultaneously and in the same direction as the cell density changes in macroscopic measurement tools. Therefore, it is worth noting that macroscopic measurements yield lower bounds of cell contractility, but microscopic measurement provides upper bounds for the applied forces. In particular, this study suggests that the maximum contractile force generated by individual fibroblasts is $\sim 200 \mathrm{nN}$.

\section{Conclusion}

We examined cell contractility on self-standing fiber scaffolds fabricated by using femtosecond laser induced two-photon polymerization. Contractile forces generated by individual cells were calculated by using columnar buckling analysis. The simple structure of the fiber scaffolds facilitated microscopic observation of cell contraction. Experimental assumptions and calculation errors could be reduced compared to previous studies. The force magnitude obtained by this investigation offers an upper bound of contractility of individual cells that can be useful in order to design bioactive scaffolds for tissue engineering, complementing the lower bound suggested by macroscopic measurement representing multiple cells within threedimensional structures. This method can also be used to understand contractile forces generated by cells in more detail with respect to wound healing, and improve contractility of cells for pathological wound healing and therapeutic strategies. 
Acknowledgment Support by the U.S. National Science Foundation under grant DMI-0556363 is gratefully acknowledged.

Open Access This article is distributed under the terms of the Creative Commons Attribution Noncommercial License which permits any noncommercial use, distribution, and reproduction in any medium, provided the original author(s) and source are credited.

\section{References}

K.A. Addae-Mensah, J.P. Wikswo, Exp. Biol. Med. 233(7), 792 (2008)

N.Q. Balaban, U.S. Schwarz et al., Nat. Cell Biol. 3(5), 466 (2001)

K.A. Beningo, Y.L. Wang, Trends Cell Biol. 12(2), 79 (2002)

K. Burridge, M. Chrzanowska-Wodnicka, Annu. Rev. Cell Dev. Biol. 12, 463 (1996)

R.R. Craig, Jr., Mechanics of materials, (John Wiley \& Sons, Inc., 1996), pp. 511-531

P. Delvoye, P. Wiliquet et al., J. Investig. Dermatol. 97(5), 898 (1991)

M. Eastwood, R. Porter et al., J. Cell. Physiol. 166(1), 33 (1996)
H.P. Ehrlich, J.B.M. Rajaratnam, Tissue Cell 22(4), 407 (1990)

T.M. Freyman, I.V. Yannas et al., Biomaterials 22(21), 2883 (2001)

C.G. Galbraith, M.P. Sheetz, Proc. Natl Acad. Sci. USA 94(17), 9114 (1997)

F. Grinnell, J. Cell Biol. 124(4), 401 (1994)

F. Grinnell, Trends Cell Biol. 10(9), 362 (2000)

B.A. Harley, T.M. Freyman et al., Biophys. J. 93(8), 2911 (2007a)

B.A. Harley, J.H. Leung et al., Acta Biomater. 3(4), 463 (2007b)

A.K. Harris, D. Stopak et al., Nature 290(5803), 249 (1981)

H. Hidai, D.J. Hwang et al., Appl. Phys. Mater. Sci. Process. 93(2), 443 (2008)

H. Hidai, H. Jeon et al., Biomed. Microdevices 11(3), 643 (2009)

J.L. Hutter, J. Bechhoefer, Rev. Sci. Instrum. 64(7), 1868 (1993)

K. Katoh, Y. Kano et al., J. Cell Biol. 153(3), 569 (2001)

S. Kawata, H.B. Sun et al., Nature 412(6848), 697 (2001)

M.S. Kolodney, R.B. Wysolmerski, J. Cell Biol. 117(1), 73 (1992)

J.L. Tan, J. Tien et al., Proc. Natl Acad. Sci. USA 100(4), 1484 (2003)

J.J. Tomasek, G. Gabbiani et al., Nat. Rev. Mol. Cell Biol. 3(5), 349 (2002)

I.V. Yannas, Tissue and organ regeneration in adults (Springer, New York, 2001), pp. 244-277

G.I. Zahalak, J.E. Wagenseil et al., Biophys. J. 79(5), 2369 (2000) 